\title{
A Vision-Based Method to Find Fingertips in a Closed Hand
}

\author{
Ankit Chaudhary*, Kapil Vatwani*, Tushar Agrawal ${ }^{*}$ and J.L. Raheja**
}

\begin{abstract}
Hand gesture recognition is an important area of research in the field of Human Computer Interaction $(\mathrm{HCl})$. The geometric attributes of the hand play an important role in hand shape reconstruction and gesture recognition. That said, fingertips are one of the important attributes for the detection of hand gestures and can provide valuable information from hand images. Many methods are available in scientific literature for fingertips detection with an open hand but very poor results are available for fingertips detection when the hand is closed. This paper presents a new method for the detection of fingertips in a closed hand using the corner detection method and an advanced edge detection algorithm. It is important to note that the skin color segmentation methodology did not work for fingertips detection in a closed hand. Thus the proposed method applied Gabor filter techniques for the detection of edges and then applied the corner detection algorithm for the detection of fingertips through the edges. To check the accuracy of the method, this method was tested on a vast number of images taken with a webcam. The method resulted in a higher accuracy rate of detections from the images. The method was further implemented on video for testing its validity on real time image capturing. These closed hand fingertips detection would help in controlling an electro-mechanical robotic hand via hand gesture in a natural way.
\end{abstract}

Keywords-Hand Shape Parameters, Fingertips Detection, Skin Filter, Natural Computing, Corner Detection

\section{INTRODUCTION}

One of the major developmental aims in the world today is the advancement of a more intuitive user interface system to replace the outdated pointing devices. Therefore, gesture recognition system actually provides an important alternative for modern user interface systems. This new user interface system is more intuitive and easier to use compared to mouse-based devices or touch pads. A primary implementation required in such an interface is the detection of hand gestures. This includes detecting various hand features, including the wrist, thumb, fingers, fingertips, palm face, and their orientation. Fingertips detection is a major part of gesture detection process. Many gestures can be recognized by changing the position of the fingertips with respect

\footnotetext{
※ The authors would like to thank the Director of Central Electronic Engineering Research Institute/Council of Scientific and Industrial Research (CEERI/CSIR), Pilani, for providing research facilities, and for his active encouragement and support.

Manuscript received October 18, 2011; accepted May 3, 2012.

Corresponding Author: Ankit Chaudhary

* Computer Vision Research Group, BITS Pilani, RJ 333031, India (\{ankitc.bitspilani, kapilv14, tusharagrawal29\}@ gmail.com)

** Machine Vision Lab, CEERI/CSIR, Pilani, RJ 333031, India ( jagdish@ceeri.ernet.in)
} 


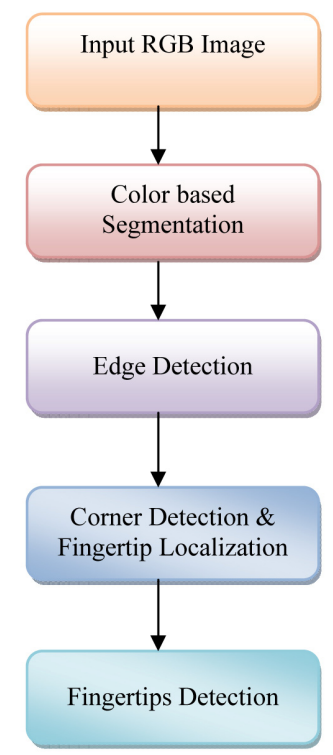

Fig. 1. Block diagram of the approach

to the base of the hand.

Enough proven scientific research is available on gesture recognition as researchers have done significant work in this area but used a wired sensor-based glove or colors to recognize hand gesture. On the other hand, this research is based on the principle of natural computing and requires nothing extra on the hand. Extensive work was done in the area of fingertips detection from still images and live video feed. This paper aims at achieving proficient detection of the fingers and fingertips in a closed hand. This paper, firstly analyzed the various existing methods for fingertip detection. Secondly, edge detection algorithms were applied onto the images to extract the Region of Interest (ROI). Our proposed method also examined a number of edge detection methods like Canny filter, Laplace filter and Gabor filter followed by Canny edge detection. The corner detection algorithm was applied to the resulting edge filtered images to separate the edges at the meeting point of two fingers. The algorithmic approach for the proposed work is shown in Fig. 1.

This research is part of our vision-based system that can be used to control a remotely located robotic hand, in which it would be able to perform the same operations as the human hand. Without wearing any mechanical electronic equipment, the user showed his hand where the palm faced the camera. The user was able to show either right or left hand to the system without restriction on the direction of the hand. Existing work about this project can be found in [1-2].

\section{RELATED WORK}

A number of methods have already been proposed for fingertips detection in an open hand. Many researchers [3-12] have used fingertips detection in their research to find information about the hand according to their applications. Some of these techniques were also applied to the 
ddclosed hand but very few achieved a greater level of accuracy. The intensity assignment model [13] first detected the orientation of the wrist and then assigned intensity values for each pixel in increasing order from the wrist to the end of the finger by proportionality. The fingertip was then detected by pixels with maximum intensity value. The contour detection method [14], however, worked by detecting the peak and valleys in the contour of the hand. The method worked fine with an open hand but did not provide accurate results with a closed hand. The pattern matching [15] technique used the Principal Component Analysis (PCA) method to extract the ROI from the image and compared to an already available database of various gestures, which helped to identify the appropriate gesture. This method offered limitations in terms of identifying the number of gestures. A survey on fingertips detection can be found in [16].

One of the methods that offered satisfactory results for hand gesture recognition was the Morphological operation based method [7, 17]. In this method, the image was pre-processed which involved background subtraction and noise removal. The background subtraction and noise removal was achieved using the morphological segmentation algorithm followed by shape decomposition and center point detection. The posture was recognized by using the orientation of the center points. The morphological methods were important tools for fingertips detection in a closed hand since these were methods that were independent of the skin color segmentation based techniques. One of the reliable methods for fingertips detection was the neural network based technique [8]. This particular method first calculated a Gabor feature vector of the whole image by applying the Gabor filter. This feature vector was then passed onto a global LLM network, which specialized in finding fingertips. This particular model claimed good results even for fingertips detection in a closed hand. An extensive study of these algorithms had given us insight into the various models implied for finger position detection. Out of all these models, the LLM neural network model claimed to detect fingers even when they were in front of the palm. However, the morphological operations based model, also claimed satisfying results. The proposed methodology works by applying the corner detection technique. The edges of the ROI, or the hand and fingers, were obtained by the application of the Gabor filter to the image in different orientation.

\section{Approach to Fingertips Detection}

The last developed system [2] was able to detect the fingertips in an open hand, but in a closed hand the last skin pixel detected in the finger's end direction, would become the fingertips. It will not detect the nails of the fingers as fingertips. The results can be seen in Fig. 2. To improve the existing system, the fingernails in the closed hand should be detected.

The presented method for fingertips detection was divided into three sections as shown in Fig. 1. The first section performed the pre-processing of the image obtained from the web camera to separate the ROI. The second section involved the application of various edge detection algorithms to the pre-processed image to separate the edges of the hand and the fingers. The final section discusses the application of the corner detection algorithms to the various edge filtered images to obtain the corners in these images. Finally, the interested corner points of fingertips were extracted and uninterested corner points were rejected using proper conditions. 


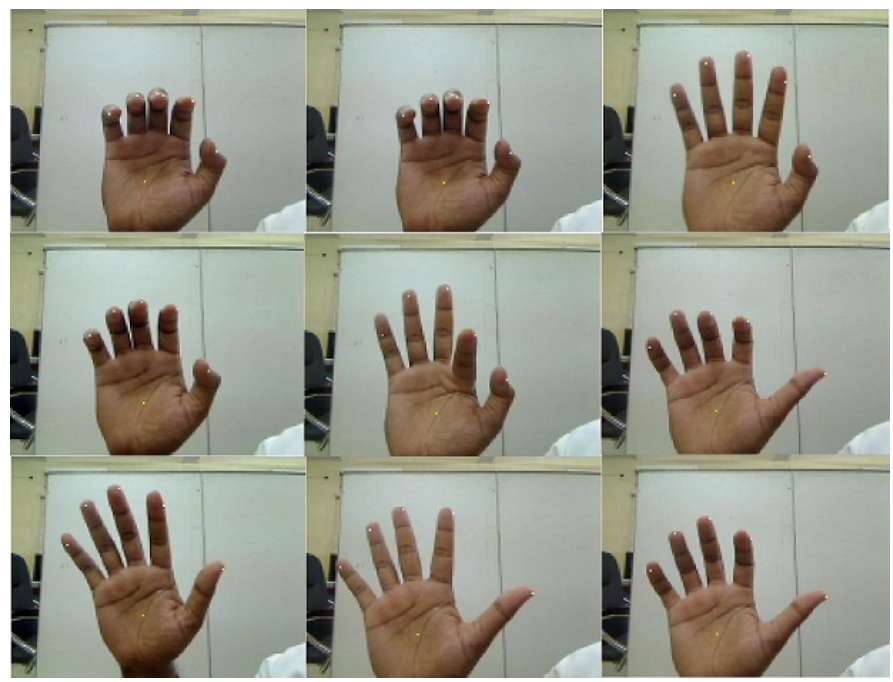

Fig. 2. Fingertips and COP detections in several hand postures [2]

\subsection{Pre-Processing}

Pre-processing was the first step in the process of the fingertips detection. It involved a number of steps to segment the region of the hand from the image. First, the area of interest was separated from the background using color differences from the background environment and background subtraction. In this case, the background environment was taken as a white wall for the initial results. The conditions for filtering the hand from background were as follows:

-Pixel's Red color should be $10 \%$ greater than its green color.

-Pixel's Red color should be 5\% greater than its blue color.

-Pixel should not have a green color and a blue color over a $60 \%$ of the maximum.

The results of the thresholding applied to some of the test cases are shown in Fig. 3.
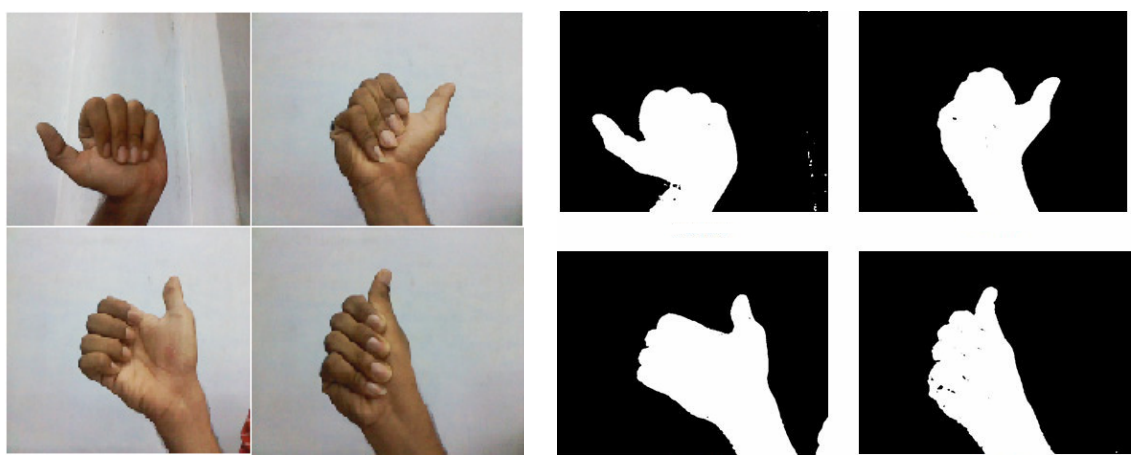

Fig. 3. Results of hand segmentation 


\subsection{Edge Detection}

There are a number of methods available for the detection of edges in an image. Three different filters were tried on the input images to detect the edges. These three methods were the Canny filter, Laplace filter and Gabor filter. The Canny edge detection filter took the derivative of an image to find the gradients and determined the direction of these gradients (vertical, horizontal, diagonal up, diagonal down). Then if the amplitude of a given gradient was high enough (the high threshold), the algorithm would trace along that gradient in its direction until the amplitude fell below the low threshold or the gradient changed direction sharply. The filter would also suppress local non-maximums around the edges. A particular set of parameters were chosen and implemented for the available test cases.

The Laplace edge detection filter was also tested on the images. The edges were clear around the hand but the filter was not able to extract the edges near the fingertips, which were the key points of interest in our corner detection algorithm. The third filter type tried on the input images was the Gabor filter. A 2D Gabor filter is represented as follows:

$$
g(x, y)=\exp \left(-\frac{x^{2}+y^{2}}{2 \sigma^{2}}\right) f\left(u_{0} x+v_{0} x\right)
$$

Where $\mathrm{f}(\mathrm{x})=\operatorname{Sin}(\mathrm{x})$ or $\operatorname{Cos}(\mathrm{x})$ and $\mathrm{x}$ is the angle. This particular function is implemented by calculating $\mathrm{g}(\mathrm{x}, \mathrm{y})$ and then applying it onto the image. The filter was applied at a different orientation to the test images. Finally, a set of parameters were fixed for the test images. One of the advantages to using the Gabor filter was that it gave enhanced edges in the direction of the angle in which the Gabor filter was applied. The application of a superposition of such Gabor filters for different orientation resulted in very good detections.

\subsection{Corner Detection and Fingertip Localization}

Corner detection involved the detection of corners with big Eigen values. The program first calculated the minimal Eigen value for every source image pixel and stored them in another image. It then performed non-maxima suppression where only local maxima in $3 \times 3$ neighborhoods remained. The next step was to reject the corners with the minimal Eigen value less than quality level, with a user-specified quality value. Finally, the function ensured that all the corners found were distanced enough from one another. This was done by considering the corners and checking that the distance between the newly considered feature and the features considered earlier was larger than minDistance (user-defined). The function therefore removed the features that were too close to the stronger features.

The accuracy of the corner detection algorithm was tested by applying it to various images such as the RGB image, Canny edge filtered image, Laplace edge filtered image and Gabor filtered image. The corner detection algorithm was initially tried on RGB images directly obtained from the camera. The corners obtained after running the corner detection algorithm also included corners in the background. The corners in the background were removed by including only those corners which were obtained from the threshold image in the ROI that was on the palm. The results are shown in Fig. 4.

Applying the corner detection algorithm directly onto RGB images yielded a large number of points which were not relevant. This problem was overcome by applying the corner detection algorithm to the hand's edges detected in the images. The edges of the hand were detected by 


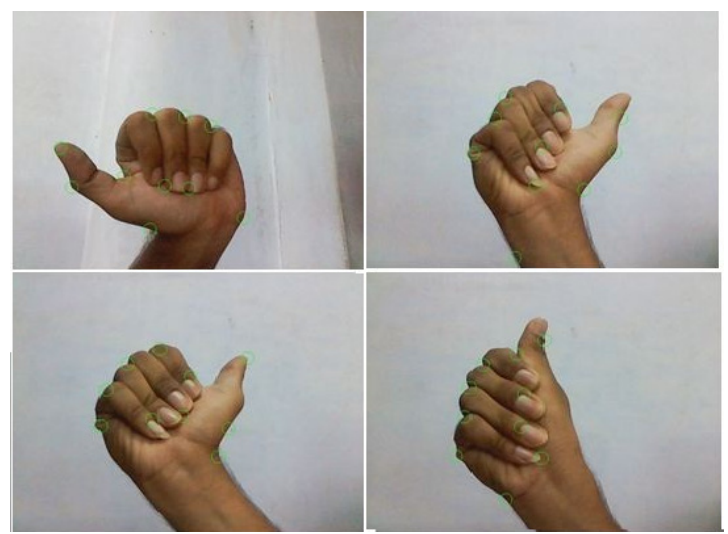

Fig. 4. Results of fingertips detection in the closed hand position from RGB images

applying the Canny edge detecting algorithm to the ROI in the gray scale image. The irrelevant points which appeared in the corner detection results of the RGB images were removed by the use of the Canny filter. The corner detection algorithm was also tested on the Laplace edge filtered images. In case of RGB images which produce more noise in preprocessing, a large number of points were detected. These points were rejected by obtaining the edges of the ROI by applying the Laplace filter. However, the edges obtained by the application of the Laplace filter were not strong enough, and thus the corner detection algorithms yielded less points of interest in the image. Fig. 5 shows these results.

The application of the corner detection algorithm to the edges of the hand helped us to remove the extra points, which we obtained after direct application to the RGB image. However, we applied the Gabor filter for better edge detection. We then applied the corner detection directly to the Gabor filtered image. Because the results were not satisfactory, we again applied Canny to

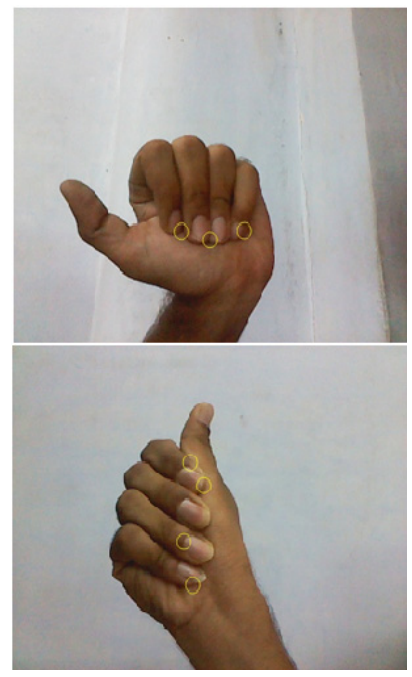

Fig. 5. Results of fingertips detection using Canny-Gabor filter 
the Gabor filtered image and then implemented the corner edge detection onto the resultant edge image. The results were quite satisfactory for an orientation of Gabor filter. Thus the quality of the results varied a lot depending on the angle of the Gabor filter, as Fig. 5 shows for 105 degrees.

\section{RESULTS}

The proposed method was implemented using OpenCV ${ }^{\circledR}$ libraries as the tools for processing the images due to their specific application to real-time image processing and flexible inclusion into a Linux C-environment running on an Intel ${ }^{\circledR}$ i5 Processor. In addition to this test, the cases used in this method were set for particular environmental parameters such as light intensity and orientation, which were decided by examining the various sample test cases. The experimental setup is shown in Fig. 6.

By using either their right or left hand, the users gave input with their hand in random directions. The system was tested in different conditions for a long time to check its robustness with different users. The system was free from the user's hand geometry and would work the same for everyone. Only Nguyen [7] work is available on closed finger detection in scientific literature and the accuracy was $10-20 \%$, while our results showed $50-60 \%$ accuracy. Nonetheless, we have been working to improve our system to make it more accurate.

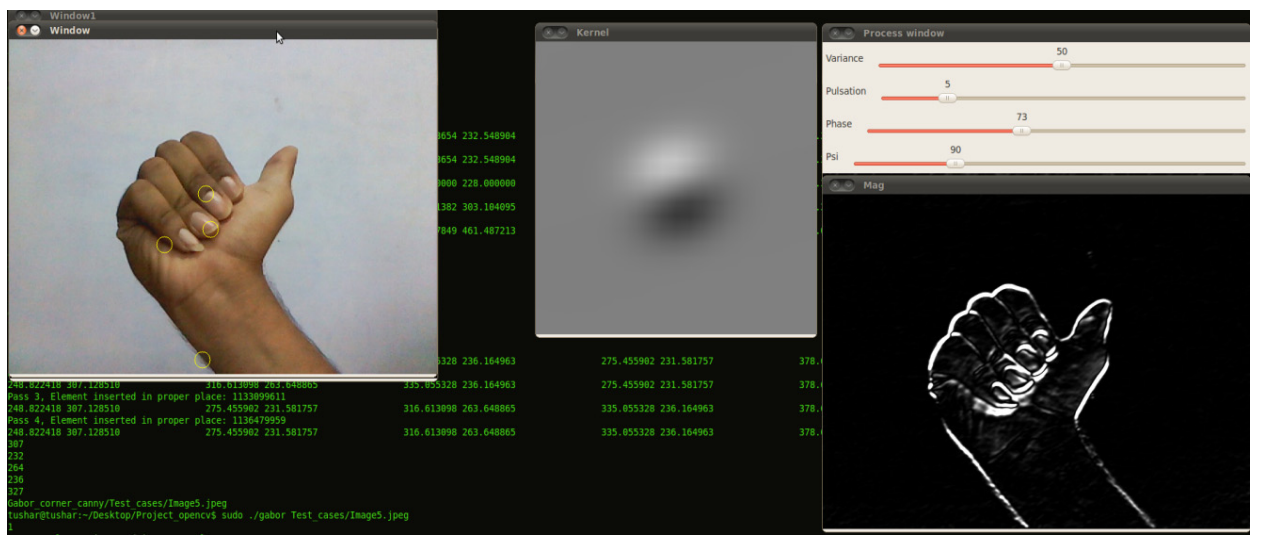

Fig. 6. Experimental setup view

\section{CONCLUSION}

After studying literature on fingertips detection, we saw that the detection of fingertips in an open hand has already been implemented using various methods. However, detecting fingertips in a closed fist remains a challenge, and the results obtained by these methods are not satisfactory.

The paper presents the use of corner detection algorithm to detect fingertips. One essential part of this method includes the reduction of noise during edge detection in an image. To achieve this goal, different filters were applied, such as the Canny filter, Laplace filter and Ga- 
bor filter. Out of these filters, the Gabor filter resulted in satisfactory results. We will try to improve the current method in our future work. In addition to this, we are working to explore morphological operations for fingertips detection, as morphological operations are less prone to noise produced in preprocessing.

\section{REFERENCES}

[1] Chaudhary, A., Raheja, J.L., Singal, K., Raheja,S., An ANN based Approach to Calculate Robotic fingers positions, Published as Book Chapter in Lecture Notes in Computer Sciences, CCIS, Springer Berlin Heidelberg, Vol.192, No.3, 2011, pp.488-496.

[2] Chaudhary, A., Raheja, J. L., Das, K., Raheja, S., A Vision based Geometrical Method to find Fingers Positions in Real Time Hand Gesture Recognition, Journal of Software, Academy Publisher, Vol.7, 2012.

[3] Gastaldi G. and et al., "a man-machine communication system based on the visual analysis of dynamic gestures”, International conference on image processing, Genoa, Italy, September, 2005, pp.397-400,.

[4] Kim, J. M., Lee, W. K., "Hand shape Recognition using fingertips", In the proceedings of fifth International Conference on Fuzzy Systems and Knowledge Discovery, Jinan, Shandong, China, October, 2008, pp.44-48.

[5] Lee, B., Chun, J., "Manipulation of virtual objects in marker-less AR system by fingertip tracking and hand gesture recognition", Proceedings of $2^{\text {nd }}$ international conference on interaction science: Information Technology, Culture and Human, Seoul, Korea, 2009, pp.1110-1115.

[6] Lee, D., Park, Y., "Vision-Based Remote Control System by Motion Detection and Open Finger Counting”, IEEE Transactions on Consumer Electronics, Vol.55, Issue 4, November, 2009, pp.23082313.

[7] Nguyen, D.D., Pham, T.C., Jeon, J.W., "Fingertip Detection with Morphology and Geometric Calculation”, IEEE/RSJ International Conference on Intelligent Robots and Systems, St. Louis, USA, October, 2009, pp.1460-1465.

[8] Nolker, C., Ritter, H., "Visual Recognition of Continuous Hand Postures", IEEE Transactions on neural networks, Vol.13, No.4, July, 2002, pp.983-994.

[9] Raheja, J.L., Chaudhary, “A., and Singal, K., Tracking of Fingertips and Centers of Palm Using KINECT", Proceedings of 3rd Internatinal Conference on Computational Intelligence, Modelling and Simulation (CIMSiM), 2011, 20-22, September, 2011, pp.248-252.

[10] Sawah, A.E. and et al., "A framework for 3D hand tracking and gesture recognition using elements of genetic programming”, Proceedings of 4th Canadian conference on Computer and robot vision, Montreal, Canada, May, 2007, pp.495-502.

[11] Shin, M. C., Tsap, L. V. and Goldgof, D. B., "Gesture recognition using bezier curves for visualization navigation from registered 3d data”, Pattern Recognition, Vol.37, Issue 5, May, 2004, pp.1011-1024.

[12] Zhou, H., Ruan, Q., A "Real-time Gesture Recognition Algorithm on Video Surveillance”, Proceedings of 8th international conference on Signal Processing, Beijing, China, Vol.3, November, 2006.

[13] Raheja, J.L., Das, K., Chaudhary, "A. An Efficient Real time method of fingertip detection", Proceeding of 7th International Conference on Trends in Industrial Measurements and Automation (TIMA 2011), Chennai, INDIA, January, 2011, pp.447-450.

[14] Malik, S. Real-time Hand Tracking and Finger Tracking for Interaction, CSC2503F Project Report.

[15] Raheja J.L., Shyam R,. Kumar U., Prasad P.B., "Real-Time Robotic Hand Control using Hand Gesture”, 2nd international conference on Machine Learning and Computing, 9-11 February, 2010, Bangalore, India, pp.12-16.

[16] Chaudhary, A., Raheja, J.L., Das, K., and Raheja, S., A Survey on Hand Gesture Recognition in context of Soft Computing,Published as Book Chapter in Advanced Computing CCIS, Springer Berlin 
Heidelberg, Vol.133, 2010, pp.46-55.

[17] Choi, J., Ko N., Ko D., "Morphological Gesture Recognition Algorithm, Proceeding of IEEE region" 10th international conference on Electrical and Electronic Technology, Coimbra, Portugal, 19-22 August, 2001, pp.291-296.

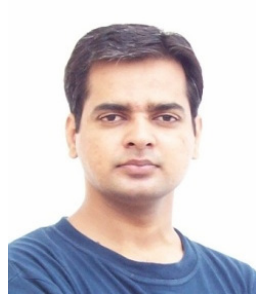

\section{Ankit Chaudhary}

He received his Master of Engineering from the Department of Computer Science, Birla Institute of Technology \& Science, Pilani, India and Ph.D. in Computer Vision, from CEERI-BITS Pilani, India. His areas of research interest are Computer Vision, Medical Imaging, Artificial Intelligence and Machine Learning. He has also worked as a system programmer at CITRIX R\&D and AVAYA INC.

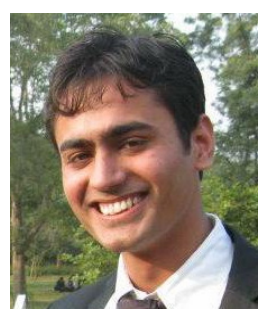

\section{Kapil Vatwani}

$\mathrm{He}$ is currently pursuing his Bachelor in Engineering from the Department of Electrical and Electronics Engineering at Birla Institute of Technology \& Science in Pilani, India, and is interested in the areas of Computer Vision and Intelligent Systems.

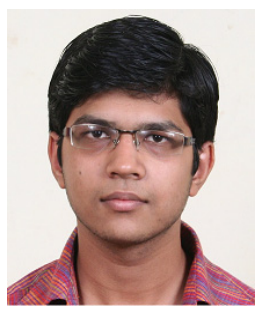

\section{Tushar Agrawal}

$\mathrm{He}$ is currently pursuing his Bachelor in Engineering from the Department of Computer Science at Birla Institute of Technology \& Science in Pilani, India, and is interested in the areas of Computer Vision and Robotics. 


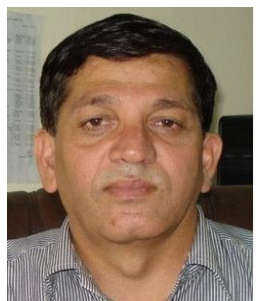

\section{J. L. Raheja}

He received his M.Tech from IIT Kharagpur, India, and Ph.D. from the Technical University of Munich, Germany. He is currently a senior scientist at CEERI, Pilani, India. His areas of research interest are Digital Image Processing, Embedded Systems and Human Computer Interface. He has been a DAAD fellow, and has published more than 80 papers for international journals and conferences. 